\section{Factores que motivan a los trabajadores en empresas de Lima Metropolitana}

\author{
Factors that motivate the workers in companies of \\ Metropolitan Lima
}

\section{RESUMEN}

El presente estudio tuvo como objetivo identificar a los factores motivadores de mayor relevancia para los trabajadores en las empresas de producción y de servicio. El diseño de investigación fue el exploratorio y descriptivo. La muestra estuvo conformada por 1,214 trabajadores de empresas de producción y de servicio de Lima Metropolitana. El instrumento utilizado para la evaluación fue el Test J\&F - Motivación Laboral (Arana, 2017) el cual presenta una confiabilidad de 0.88 . Los resultados evidencian que los factores motivacionales más relevantes para los trabajadores son: el crecimiento, el sueldo, el reconocimiento, la responsabilidad, el logro y le siguen otros factores como: el trabajo mismo, la relación con el jefe y las relaciones con los compañeros. En consecuencia, se concluye que, desarrollar los factores internos son los más relevantes para los trabajadores a la hora de identificar sus motivaciones en la empresa, incluso por encima de los factores externos.

Palabras claves: Motivación laboral; crecimiento personal; sueldo; reconocimiento; responsabilidad; logro; trabajo; relación entre compañeros; vida personal; status; seguridad; poder.

\begin{abstract}
The objective of this study was to identify the most relevant motivating factors for workers in production and service companies. The research design was exploratory and descriptive. The sample consisted of 1,214 workers from production and service companies in Metropolitan Lima. The instrument used for the evaluation was the J \& F Test - Labor Motivation (Arana, 2017) which has a reliability of 0.88 . The results show that the most relevant motivational factors for workers are: growth, salary, recognition, responsibility, achievement and other factors such as: work itself, the relationship with the boss and relations with colleagues. Consequently, it is concluded that, developing internal factors is the most relevant for workers when it comes to identifying their motivations in the company, even above external factors.

Keywords: Work motivation; personal growth; salary; recognition; responsibility; achievement; work; peer relationship; personal life; status; security; power.
\end{abstract}

\section{Walter Arana Mayorca ${ }^{1}$} walter.arana@unmsm.edu.pe Universidad Nacional Mayor de San Marcos

\footnotetext{
1 ORCID: 0000-0003-0700-4322
}

(C) Los autores. Este artículo es publicado por Gestión en el Tercer Milenio de la Facultad de Ciencias Administrativas de la Universidad Nacional Mayor de San Marcos. Este es un artículo de acceso abierto, distribuido bajo los términos de la licencia Creative Commons Atribucion - No Comercia_Compartir Igual 4.0 Internacional. (http://creativecommons.org/licenses/by-nc-sa/4.0/) que permite el uso no comercial, distribución y reproducción en cualquier medio, siempre que la obra original sea debidamente citada. 


\section{INTRODUCCIÓN}

En el mundo moderno con una economía globalizada, para que las empresas puedan lograr su permanencia en el mercado, los directivos de la empresa a desplegar todos sus esfuerzos para ser cada vez mejores y competitivos. El mejoramiento de la calidad del producto o servicio, pasa necesariamente por capitalizar las competencias del potencial humano. En consecuencia, una organización tendrá éxito o no dependiendo de la calidad de su gente.

Entre otros aspectos, saber motivar al personal es una tarea complicada, pero de singular trascendencia para lograr los objetivos empresariales y satisfacer las expectativas de los trabajadores. Entonces, la tarea es: ¿cómo motivar a los trabajadores? La explicación se inicia entendiendo que la palabra motivación proviene del latín motivus o motus, que significa causa del movimiento. En consecuencia, lo primero que se debe hacer es identificar aquellos factores que hace que la persona se mueva o actúe, conocer sus necesidades, expectativas y metas personales, para conjugar con los objetivos empresariales. Indudablemente, el factor humano es el artífice del desarrollo de toda organización.

Robbins y Judge (2017) definen la motivación como los procesos que inciden en la intensidad, dirección y persistencia del esfuerzo que realiza una persona para alcanzar una meta. La intensidad se refiere a la cantidad de esfuerzo que hace una persona orientado hacia una determinada dirección para cumplir objetivos y mantener tal actitud es la persistencia.

Precisamente, la investigación que se presenta tiene como objetivo general identificar a los factores motivadores e higiénicos de mayor relevancia para los trabajadores en las empresas de producción y de servicio. Los objetivos específicos son: a) identificar a los factores motivadores e higiénicos de mayor relevancia para los trabajadores, según el tipo de empresa; b) identificar a los factores motivadores e higiénicos de mayor relevancia para los trabajadores, según el género; c) identificar a los factores motivadores e higiénicos de mayor relevancia para los trabajadores, según las edades; y d) Identificar si los factores intrínsecos tienen mayor relevancia para los trabajadores en comparación con los factores extrínsecos.
Para motivar a una persona se debe generar determinadas condiciones que le estimule $u$ oriente a comportarse de una determinada manera. En el ámbito organizacional, la motivación debe estar orientada a que los trabajadores ejecuten sus tareas con satisfacción para el logro de los objetivos organizacionales y, por otro lado, estas condiciones generadoras de conductas satisfactorias deben estar encaminada a que el trabajador también logre lo que le interesa.

Uno de los problemas que afronta la implementación de un programa motivacional es que generalmente se desarrollan como si todos los trabajadores tuvieran las mismas motivaciones o expectativas. Se dejar de lado que todas las personas son diferentes, desean cosas distintas, les satisfacen y motivan cosas diferentes. Por lo tanto, en primera instancia, es importante identificar aquellos factores que realmente interesa o motivan a los trabajadores. Por ejemplo, muchos considerar mejorar el salario es motivante para elevar la productividad; sin embargo, Jeske (2018) considera que existen otras maneras de pensar en el empleo que se mueve más allá de los ajustes del salario.

Della Vigda y Pape (2016), en una investigación concluyen que los incentivos monetarios tienen mayor efecto motivador que los motivadores psicológicos. Pero esto no es definitivo, por cuanto Florentine (2016) en una investigación encontró que, de una lista de factores, el reconocimiento verbal de un supervisor realizado en persona es para el trabajador lo más importante. El reconocimiento del supervisor vía correo electrónico ocupa el segundo lugar. Esto revela que las personas quieren saber que sus jefes se preocupan por ellos.

Respecto a la importancia de los factores motivacionales en las organizaciones, Whetten y Cameron (2016) señalan:

\footnotetext{
"el conocimiento de las teorías motivacionales ayuda a los directivos a comprender si las recompensas organizacionales son reforzadores valiosos para individuos concretos. En la práctica, esto significa que los directivos tienen que entender qué es lo que motiva a cada uno de sus subalternos".
}

Dado que cada persona tiene sus propias expectativas y motivaciones que le interesa de sobremanera. Ivancevich, Konopaske y Matteson (2015) señalan que a cada persona le at- 
rae cierto conjunto de metas; por lo tanto, si un administrador pretende orientar el comportamiento del trabajador debe conocer algo sobre las metas del trabajador y sobre las acciones que éste emprenderá para lograrlas. El psicólogo Herzberg realizó una investigación para responder la siguiente pregunta ¿Qué espera la gente de su trabajo?

Frederick Herzberg y los factores intrínsecos y extrínsecos

En 1966 Herzberg y sus colaboradores Mausner y Snyderman realizaron una investigación basados en una muestra de 200 personas entre ingenieros y contadores. Los resultados de la investigación concluyen que hay: a) factores motivadores que producen satisfacción en el trabajo y b) factores higiénicos que evitan la insatisfacción laboral.

Los factores motivadores, llamados también satisfactores o intrínsecos, están directamente relacionados con el quehacer del trabajador; es decir, depende directamente de la actitud del trabajador frente a la tarea que ejecuta. Estos son: necesidades de logro, el reconocimiento, trabajo en sí mismo, la responsabilidad, promoción y crecimiento. Estos factores son motivadores y genera satisfacción por la labor que se realiza. Entonces, un trabajador adecuadamente motivado estará satisfecho y predispuesto a optimizar su productividad.

Los factores higiénicos, llamados también insatisfactores o extrínsecos, son aquellos factores que son administrados por los directivos de la empresa; es decir, no dependen del propio trabajador, sino de las condiciones externas que rodea a las personas en el ámbito organizacional. Estos factores higiénicos son: políticas y administración, supervisión, relación con el supervisor, condiciones de trabajo, sueldo, relación con los compañeros, vida personal, relación con los subordinados, estatus y seguridad. El papel de estos factores es impedir que se produzca la insatisfacción entre los trabajadores. Sin embargo, la estimulación o la presencia de estos factores externos no provocaran satisfacción laboral, según la concepción de Herzberg.

La relación entre los factores intrínsecos e higiénicos es que los primeros se constituyen como la energía, el impulso que hace que las per- sonas se dirijan hacia un determinado objetivo, en tanto los segundos se constituyen como el soporte en la que se apoyan los primeros. Es decir, ambos deben coexistir en una óptima gestión.

\section{MÉTODOS}

El enfoque de la investigación es cuantitativo dado que los resultados se expresan en valores numéricos. Es de tipo exploratorio por tratarse de un tema poco investigado y es descriptivo porque se presentan los resultados obtenidos. El diseño es no experimental.

La población encuestada está constituida por 1,214 trabajadores de diversas empresas de Lima. El muestreo para el estudio es no probabilístico de tipo intencional, dado que los encuestadores consideraron la accesibilidad a la muestra de investigación en sus respectivos centros de trabajo. El trabajo de campo fue realizado por los alumnos de la Maestría en Dirección de Recursos Humanos de la Unidad de Posgrado de la Facultad de Ciencias Administrativas de la Universidad Nacional Mayor de San Marcos, durante el primer y segundo semestre de 2017.

En la muestra han participado 999 (82.29\%) trabajadores de empresas de servicios y 215 (17.71\%) de producción. Respecto al género, 641 fueron hombres y 573 mujeres. Participaron 445 trabajadores menores de 30 años de edad, 596 de 31 a 45 años de edad y 173 de 46 a más años de edad.

En la investigación se utilizó el Test J\&F Motivación Laboral, elaborado por Walter Arana, que permite identificar y medir los factores motivacionales de los trabajadores. Dicho test tiene como índice de validez de contenido $=$ 0,88 . La validez de constructo $=0,97$. Los resultados de la confiabilidad fueron: logro ( $\mathrm{r}=$ $0,87)$, reconocimiento $(r=0,81)$, trabajo mismo $(r=0,91)$, responsabilidad $(r=0,90)$, crecimiento $(r=0,94)$, política y administración $(r=0,57)$, supervisión $(r=0,55)$, relación con el jefe $(r=$ $0,81)$, condiciones de trabajo $(r=0,76)$, remuneraciones $(r=0,94)$, relaciones con los compañeros $(r=0,88)$, vida personal $(r=0,55)$, relación con los subordinados $(0,15)$, estatus $(\mathrm{r}=$ $0,40)$, seguridad $(r=0,96)$ y poder $(r=0,12)$, Los factores cuyo grado de confiabilidad fue menor a $r=0,57$, fueron excluidos del test por tener moderada o baja confiabilidad. 


\section{RESULTADOS}

Luego de la aplicación del Test J\&F - Motivación Laboral, se ha obtenido los siguientes resultados, respecto al grado de importancia que conceden los trabajadores:

1. Según el orden de importancia, el $18,23 \%$ de los trabajadores consideran que el factor que más les motiva es el crecimiento, seguido por el sueldo con $16,11 \%$, reconocimiento $10,27 \%$, responsabilidad 9,38\%, logro 9,17\%, el trabajo mismo 8,25\%, relación con el jefe 7,64\%, relaciones con los compañeros $7,58 \%$, seguridad (6,96\%) y condiciones de trabajo (6,41\%). Los resultados respecto a la incidencia de los otros Factores motivacionales en el trabajo se describen en la figura 1.

2. Según el tipo de empresa, el $17,64 \%$ de los trabajadores de empresas de producción valoran al factor crecimiento como principal elemento motivante en comparación del $18,35 \%$ de los trabajadores de empresas de servicio. El $17,23 \%$ de los trabajadores de empresas de producción y el $15,88 \%$ de los trabajadores de empresas de servicios valoran en segunda instancia de importancia al sueldo. Los resultados de los otros factores motivantes e higiénicos, se describe en la figura 2 .

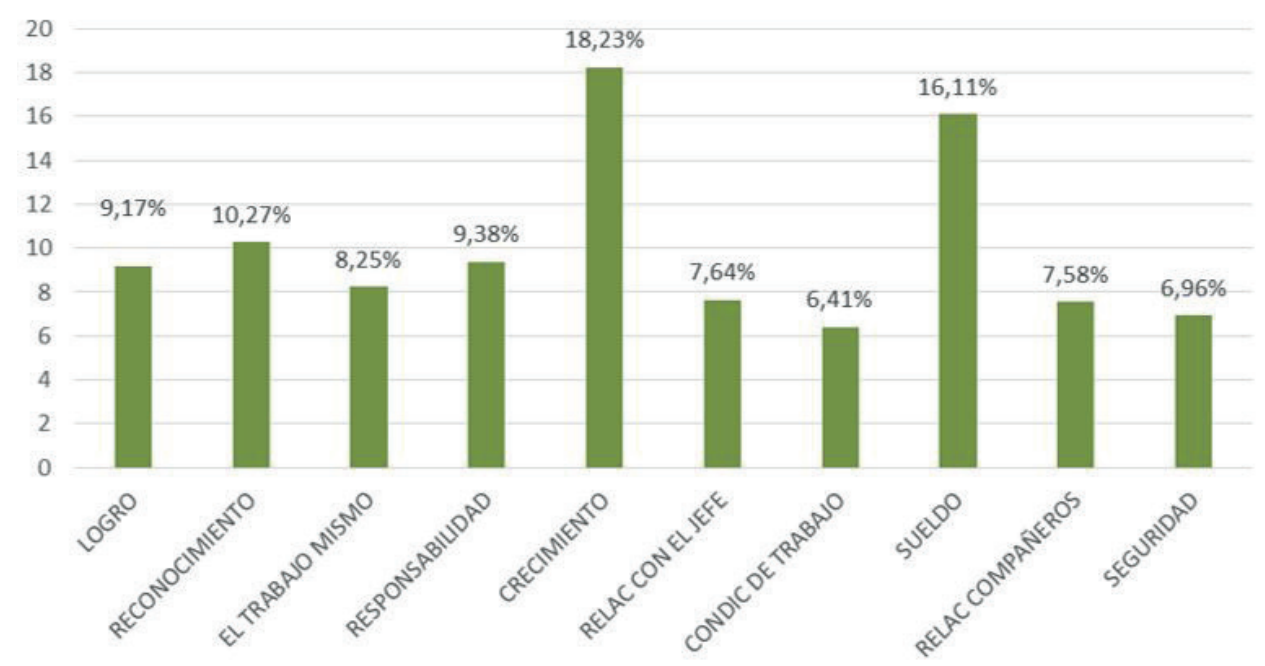

Figura 1. Factores motivacionales en el trabajo

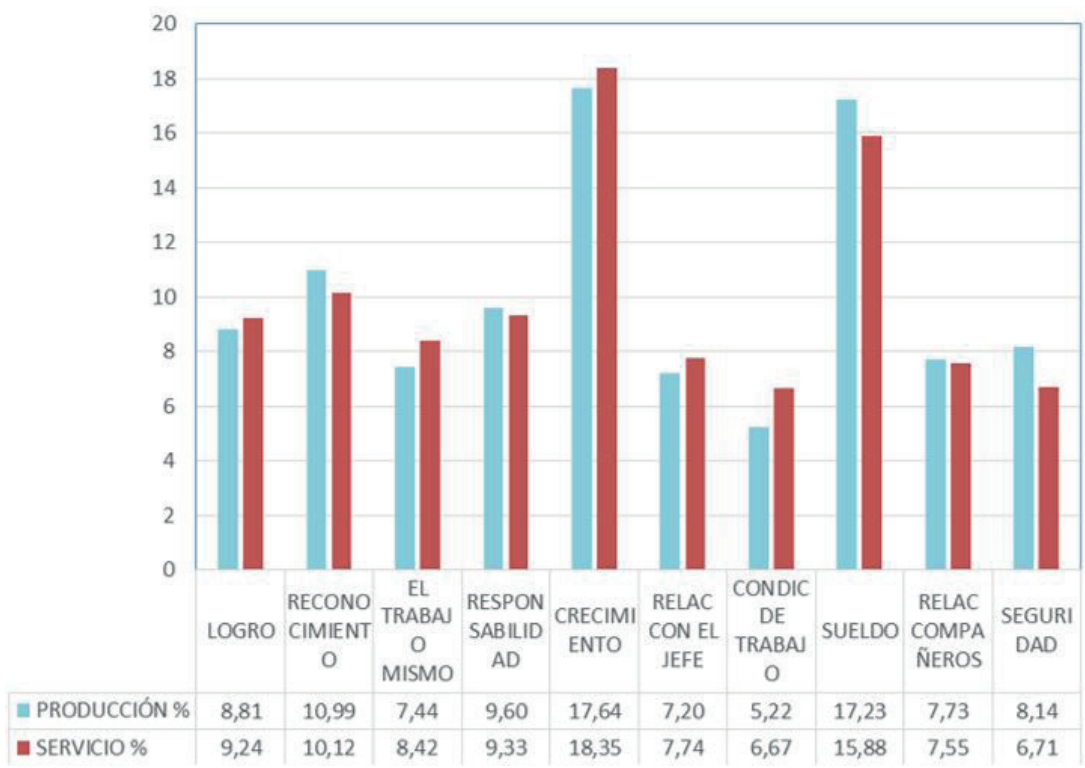

Figura 2. Factores motivacionales a los trabajadores según tipo de empresa 
3. Según el género de los trabajadores, el $18,18 \%$ del personal femenino valoran al factor crecimiento como principal elemento motivante en comparación del 18,27\% del género masculino. Los resultados de los otros factores describen en la figura 3 .
4. Según la edad, los trabajadores menores de 30 años de edad valoran al crecimiento en un $18,70 \%$, en $18,06 \%$ quienes tienen de 31 a 45 años de edad y 17,60\% los mayores de 46 años de edad. Los resultados de los otros factores se describen en la figura 4 .

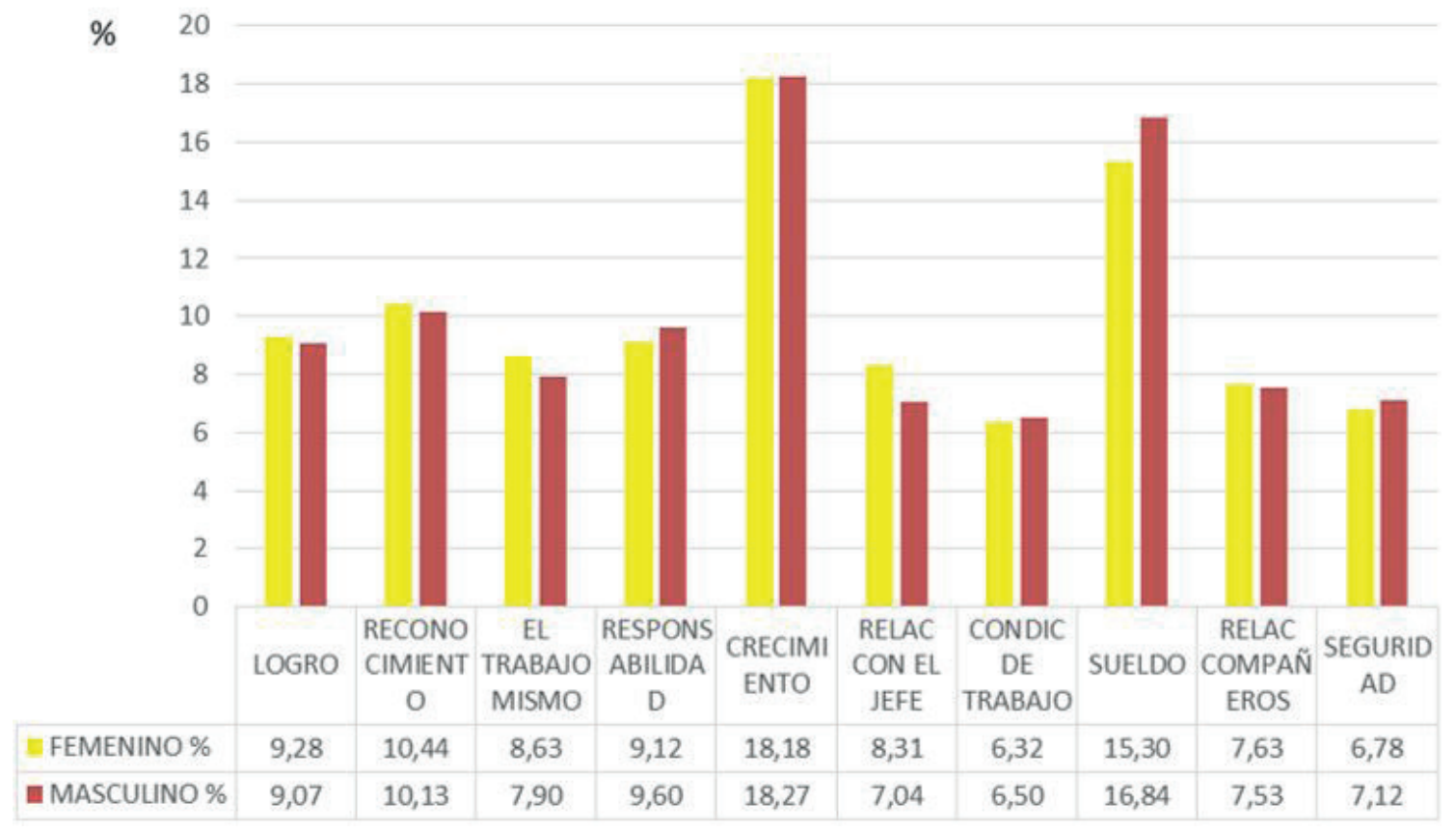

Figura 3. Factores que motivan a los trabajadores según género

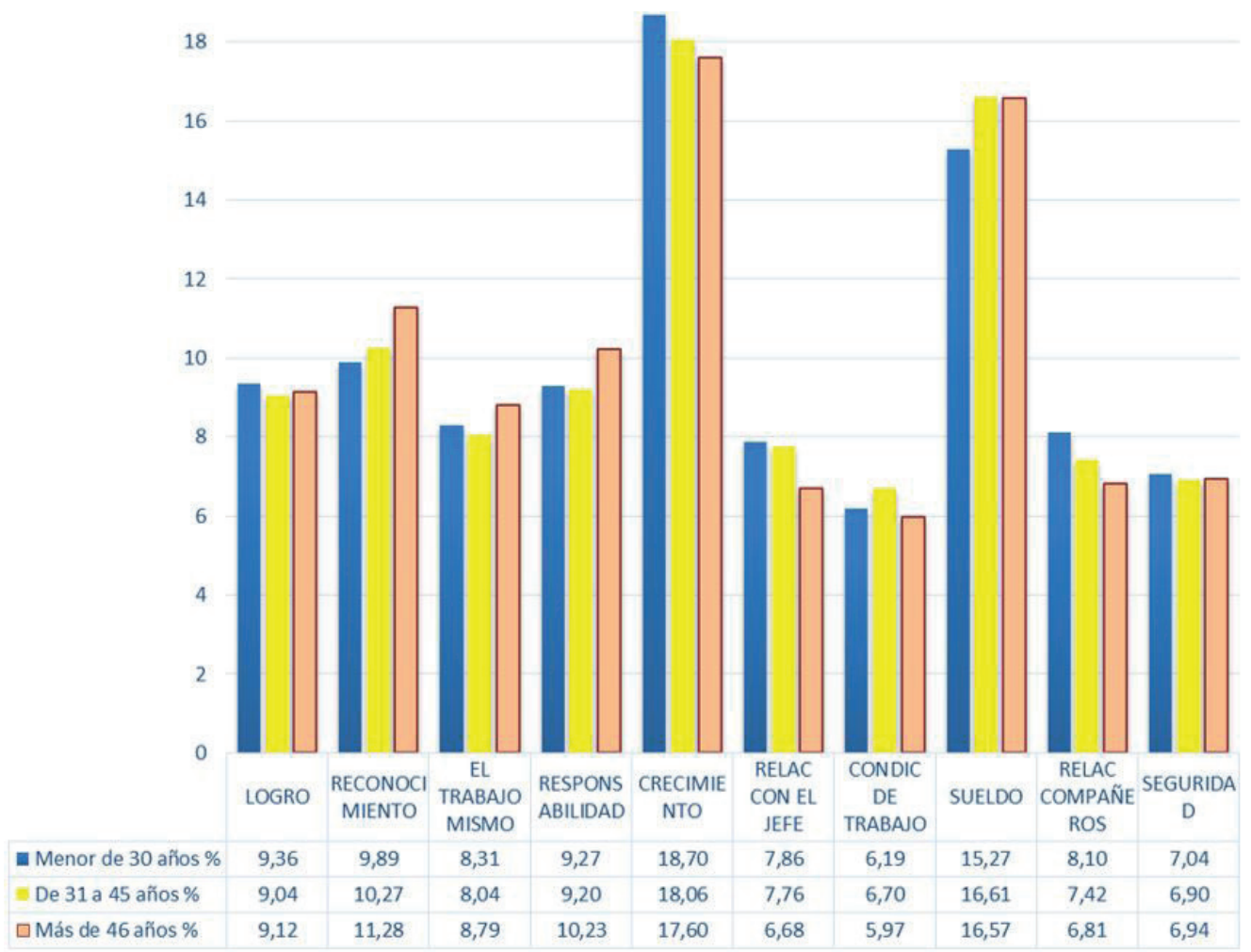

Figura 4. Factores que motivan a los trabajadores según edades 


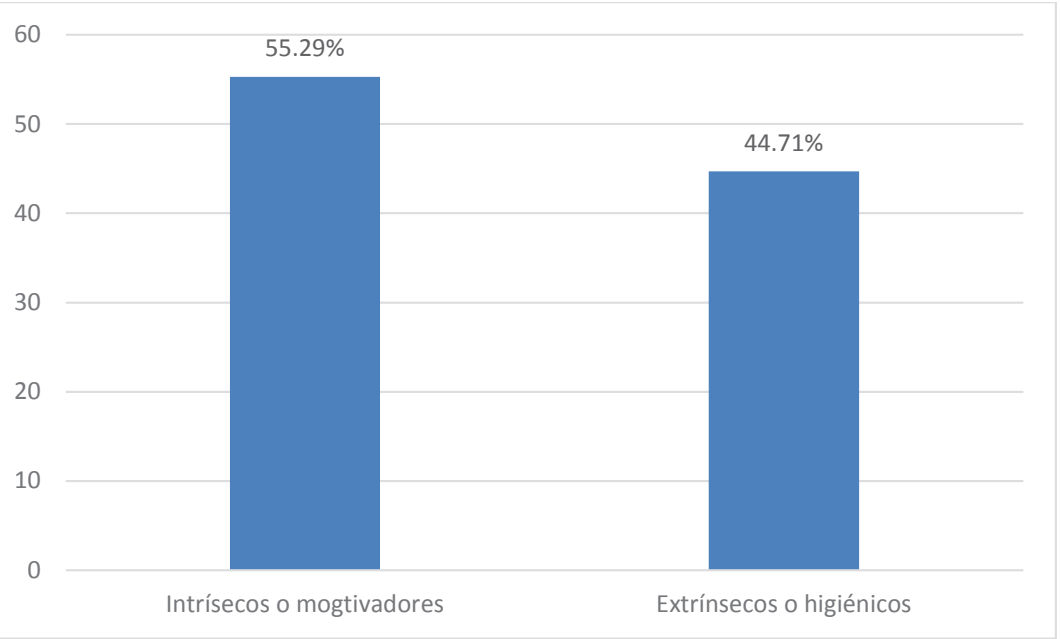

Figura 5. Relevancia de los factores según los trabajadores

5. De manera general, a los trabajadores les motiva los factores intrínsecos (necesidades de logro, reconocimiento, trabajo en sí mismo, responsabilidad y crecimiento) en un $55,29 \%$ en comparación al 44,71\% de los factores extrínsecos o higiénicos (relación con el jefe, condiciones de trabajo, sueldo y seguridad), tal como se aprecia en la figura 5 .

\section{DISCUSIÓN}

Según los resultados de la aplicación del Test J\&F - Motivación Laboral, de los 10 factores evaluados a 1,214 trabajadores, el 18,23\% de los trabajadores consideran que el factor que más les motiva es el crecimiento, seguido por el sueldo con $16,11 \%$, reconocimiento $10,27 \%$, responsabilidad 9,38\%, logro 9,17\%, el trabajo mismo $8,25 \%$, relación con el jefe 7,64\%, relaciones con los compañeros $7,58 \%$, seguridad $(6,96 \%)$ y condiciones de trabajo $(6,41 \%)$.

Se realizó una investigación en George Mason University, cuyos resultados provienen de un estudio de 1,000 empleados (Whetten y Cameron, 2016). En dicho estudio se evaluó 10 componentes, donde el factor crecimiento ocupa el sexto lugar y el sueldo quinto lugar. En este estudio concluyen que los tres factores de mayor relevancia son: trabajo interesante, reconocimiento pleno del trabajo realizado y sensación de estar enterado de todo. Los dos factores de menor preferencia son: ayuda comprensiva para los problemas personales y disciplina moderada.
Teniendo en cuenta el género de los trabajadores, en la investigación realizada mediante el Test J\&F - Motivación Laboral, el 18.18\% del personal femenino valoran al factor crecimiento como principal elemento motivante en comparación del $18.27 \%$ del género masculino. En el estudio realizada en George Mason University (citado en Whetten y Cameron, 2016), el crecimiento ocupa el sexto lugar en ambos géneros, siendo el trabajo interesante el de mayor relevancia.

Según el Test J\&F - Motivación Laboral, teniendo en cuenta la edad de los trabajadores, los menores de 30 años de edad valoran al crecimiento en un $18.70 \%$, en $18.06 \%$ quienes tienen de 31 a 45 años de edad y $17.60 \%$ los mayores de 46 años de edad. En el estudio realizado en George Mason University, el crecimiento, según los menores de 30 años ocupa el tercer lugar de preferencia, de 31 a 40 años de edad consideran en sexto lugar y de 41 a 50 años de edad consideran en octavo lugar.

El 2010, González, Sánchez y López-Guzmán, realizaron el estudio sobre satisfacción laboral como factor crítico para la calidad - El caso del sector hostelero de la provincia de Córdoba - España, cuya muestra estuvo conformada por 525 trabajadores y utilizaron la escala de Likert para evaluar 14 factores. La mayor valoración está referida a la relación con los compañeros (4,12 d promedio). El factor posibilidad de desarrollo (crecimiento) esa ubicada en el noveno lugar en el orden de importancia con 3,32 de promedio. La remuneración ocupa el treceavo lugar de importancia (2,76 de promedio). 
Según la investigación realizada por Goal Manager Employee Motivation Survey ante la pregunta ¿Qué te gusta de tu trabajo actual? La mayoría de los entrevistados, según orden de importancia, prefieren a las personas y ambiente de trabajo, seguido por las buenas prácticas directivas, trabajo que plantea retos, flexibilidad, salario, autonomía, oportunidad de capacitación y los de menor relevancia son: acciones, tecnología y trabajo en equipo. Ivancevich, Konopake y Matteson (2015).

Great Place to Work $®$ ha publicado en el portal Info Capital Humano, una encuesta realizada a más de 2.000 personas en el Perú. De los 6 factores evaluados (los beneficios de mi empresa, mi empresa me retribuye los logros, mi familia, mi jefe, mi sueldo y nada, me siento desmotivado), la familia, ocupa el primer lugar $(50 \%)$ como factor motivante para hacer mejor su trabajo.

Gherman, Iturbe y Osorio Murillo (2011) realizaron la tesis: La teoría motivacional de los dos factores: un caso de estudio, con un enfoque cualitativo a fin de comparar los resultados obtenidos por Herzberg y trabajadores peruanos (472) del sector retail. Los resultados de dicho estudio señalan que los factores motivadores, según el orden de importancia, son los siguientes: 1 . Relaciones interpersonales con supervisores o jefes, 2 . Relaciones interpersonales con colegas, 3. Reconocimiento, 4. Política y dirección de la empresa, 5. El trabajo mismo, 6. Posibilidad de crecimiento, 7. Logro, g) Responsabilidad y 8. Promoción. Con relación a los factores higiénicos, según el orden de importancia son: 1. Condiciones de trabajo, 2. Salario o remuneración, 3. Supervisión técnica y 4 . Vida personal.

Oliveros y Torres (2016), realizaron la investigación sobre Factores motivacionales hacia el trabajo en el departamento de enfermería de un hospital de cuarto nivel en la ciudad de Bogotá. Los resultados de la aplicación del CMT creado por Fernando Toro, concluyen que con relación a los factores intrínsecos se encontró que el 6,6\% de las personas encuestadas se encuentran en un nivel alto, el $89,7 \%$ en un nivel medio y el 3,7\% en un nivel bajo. Por otro lado, al especificar los factores motivacionales se ha encontrado que el $30,7 \%$ de los encuestados tienen un nivel bajo, el $38,9 \%$ un nivel medio y el 30,4\% un nivel alto. Con relación a la categoría condiciones motivacionales externas es bajo con un $22,5 \%$, medio con un $33,9 \%$ y alto con un $42,6 \%$.

Los resultados de la aplicación del Test J\&F - Motivación Laboral, de manera general, concluye que los factores relevantes para los trabajadores, según el orden de importancia son: los factores intrínsecos o motivadores con 55.29\% de preferencia, en comparación al $44.71 \%$ de los factores extrínsecos o higiénicos. Este resultado es similar a lo encontrado en una investigación realizada por Sdrali, Goussia-Rizou, Giannouli y Makris (2016), en la que concluyen que las fuerzas intrínsecas motivan a los empleados en mayor grado, sin que esto signifique la no importancia del aspecto financiero.

\section{CONCLUSIONES}

Para los trabajadores, los factores intrínsecos o motivadores son de mayor relevancia en comparación a los factores extrínsecos o higiénicos. Similar resultado halló Sdrali, Goussia-Rizou, Giannouli y Makris (2016).

Entre los factores intrínsecos o motivadores, el crecimiento ocupa el mayor porcentaje $(18,23 \%)$ de preferencia. En la investigación citada por Whetten y Cameron (2016), el factor crecimiento ocupa el sexto lugar de importancia. En otra investigación realizada en Córdova - España, Gonzáles, Sánchez y López-Guzmán (2010), de 14 factores estudiados, encontraron que el factor posibilidad de desarrollo ocupa el noveno lugar de importancia. Gherman, Iturbe y Osorio Murillo (2011), en Lima encontraron que, de nueve factores, el crecimiento ocupa el sexto lugar.

De los factores extrínsecos o higiénicos, el sueldo es el más relevante con $16.11 \%$ de preferencia. En la investigación citada por Whetten y Cameron (2016), el factor sueldo ocupa el quinto lugar de preferencia. Gonzáles, Sánchez y López-Guzmán (2010), en Córdova - España, de 14 factores estudiados, encontraron que el factor las remuneraciones ocupa el treceavo lugar de importancia. Gherman, Iturbe y Osorio Murillo (2011), en Lima, hallaron que entre los cuatro factores higiénicos, la remuneración o salario ocupa el segundo lugar de importancia. 
Las evidencias demuestran que si bien los factores intrínsecos o motivadores (55.29\%) son de mayor relevancia para los trabajadores en comparación con los factores extrínsecos o higiénicos (44.71\%), estas diferencias son relativamente significativas. Hay estudios que demuestran que el crecimiento no tiene tanta relevancia como se ha obtenido en la presente investigación. Es probable que existan otros factores macros de carácter social o económico, que condiciona la decisión de los trabajadores respecto a los factores que les motivan en el ámbito laboral.

\section{REFERENCIAS}

DellaVigna, S., \& Pope, D. (2016). What motivates effort? evidence and expert forecasts. Cambridge: National Bureau of Economic Research, Inc. doi:http://dx.doi.org/10.3386/w22193

Florentine, S. (2016). What really motivates workers? (it's not always money). Cio, Retrieved from https://search.proquest.com/ docview/1784661985?accountid=12268

Gherman, T., Iturbe, J. \& Osorio, D. (2011). La teoría motivacional de los dos factores: un caso de estudio. (Tesis de Maestría). Lima: Pontificia Universidad Católica del Perú.

González, F., Sánchez S. y López-Guzmán, T. (2010). Satisfacción laboral como factor crítico para la calidad: El caso del sector hostelero de la provincia de Córdoba - España. Estudios y perspectivas en turismo, 20(5), 1047-1068. Recuperado en 10 de agosto de 2017, de http://www.scielo.org. ar/scielo.php?script=sci_arttext $\&$ pid=S1851$17322011000500005 \& \operatorname{lng}=\mathrm{es} \& \operatorname{tlng}=\mathrm{es}$.
Herzberg, F. (1966). Work and nature of man, World Publishing Company.

Info Capital Humano (2017). El 70\% de los peruanos no está contento con su trabajo. Recuperado de http://www.infocapitalhumano.pe/recursos-humanos/noticias-y-movidas/el-70-de-losperuanos-no-esta-contento-con-su-trabajo/

Ivancevich, J., Konopaske, R. y Matteson, M. (2015). Comportamiento organizacional. (séptima Edición). México Mc Graw Hill.

Jeske, C. (2018). Why work? do we understand what motivates work-related decisions in south africa? Journal of Southern African Studies, 44(1), 27-42. doi:http://dx.doi.org/10.1080/0305707 0.2018 .1403219

Oliveros, J., Fandiño, J. y Torres, Y. (2016). Factores motivacionales hacia el trabajo en el departamento de enfermería de un hospital de cuarto nivel en la ciudad de Bogotá. Recuperado de http://ac.els-cdn.com/S0121737216300279/1s2.0-S 0121737216300279 - main.pdf? tid $=0$ b $87 a 43 e-7 e 14-11$ e7-acde-00000aacb361\&acdnat $=1502401201 \_16 \mathrm{c} 0 \mathrm{~b} 1 \mathrm{~d} 5 \mathrm{cb}-$ 23f37e0229fe06a7d73b39

Robbins, S. y Judge, T. (2017). Comportamiento organizacional. (17ma. Ed.). México: Pearson.

Sdrali, D., Goussia-Rizou, M., Giannouli, P., \& Makris, K. (2016). What motivates employees to engage in the social economy sector? A case study of greek cooperative enterprises. International Journal of Social Economics, 43(12), 1334-1350. Retrieved from https://search.proquest.com/ docview/1842865729?accountid=12268

Whetten, D. y Cameron, K. (2016). Desarrollo de habilidades directivas. México: Pearson Educación. 